\title{
Community Dynamics in Smart City Digital Twins: A Computer Vision-based Approach for Monitoring and Forecasting Collective Urban Hazard Exposure
}

\author{
Dimitrios Mavrokapnidis \\ Georgia Institute of Technology \\ dimavrok@gatech.edu
}

\author{
Neda Mohammadi \\ Georgia Institute of Technology \\ nedam@gatech.edu
}

\author{
John E. Taylor \\ Georgia Institute of Technology \\ jet@gatech.edu
}

\begin{abstract}
Urbanization and the growth of human population are leading to increased complexities in the interactions of citizens with public spaces, creating cities that must be more responsive to community dynamics. Despite the critical need for community-based city management, current decision-making approaches are often uninformed by the collective behavior of communities across time and in different locations. Here, we introduce a computer vision-based monitoring and forecasting approach for Smart City Digital Twins (SCDT) that enables integrating collective behavior into the spatiotemporal assessments of exposure to urban heat stress. Our results from a pilot case study in the city of Columbus, GA, demonstrate the significance of integrating community dynamics into monitoring and forecasting urban hazard exposure. This ongoing study highlights how SCDT can make community dynamics more accessible to and responsive by city managers.
\end{abstract}

\section{Introduction}

The world's population is projected to rise from 7.7 billion in 2019 to 9.7 billion people in 2050, with more than $66 \%$ residing in urban areas [1]. In the next decade, one in four people worldwide will live in a city with more than 1 million inhabitants [2]. This rapid urbanization is continuously adding more complexity to human interactions with the built environment, making cities increasingly governed by community dynamics, vulnerable to environmental threats, less reactive to emergency events, and, consequently, jeopardizing human lives [3]. To address these challenges, technological advancements in cloud computing, big data analytics, and the Internet of Things (IoT) are increasingly adopted by city managers and policymakers with the goal of creating smarter and more connected communities [4]. However, these recent trends often lead to smart city products and interventions that neglect the human factor and the ultimate goal of assessing and improving quality of life for a city's inhabitants [5]. Incorporating citizens, stakeholders, and overall community dynamics in urban decision making together with smart city technologyenabled visual feedback [6] can foster healthier, safer, and more livable communities.

Smart City Digital Twins (SCDT), defined as digital replicas of cities continuously enriched by spatiotemporal data stemming from human and infrastructure systems [7], aim to transform the emerging challenges of urbanization into opportunities for higher quality of life through citizen-centric city management. Enabled by the IoT capabilities, and by virtually engaging users through immersive reality, digital twins are designed to capture and visualize human-infrastructure interactions, predict future behaviors, test what-if scenarios, and, thus, reify proactive city management. Monitoring human behavior and interactions in the urban context constitutes a critical step towards this realization. SCDT have previously captured human behavior and interactions in cities using social media [8] and crowdsourcing data through individual mobile phones [9]. Largely due to data limitations, these methods are limited in their ability to locate and capture the collective interactions of different communities.

Recent advancements in computer vision and deep learning have made substantial contributions to the field of urban analytics, providing cities with a new set of machine eyes, able to perceive urban interactions between humans, vehicles, and infrastructure [10]. Current research efforts in this area, however, have primarily focused on investigating the urban environment during pre-determined periods [10], and thus, are unable to continuously monitor and predict the hazardous implications of the ongoing urbanization. Moreover, many computer vision-based studies are problem-specific (e.g., focused on pedestrian safety [11], traffic monitoring [12], etc.) and, thus, the results are not consistently interoperable with, and 
generalizable to other, smart city applications. Those IoT-based testbeds for smart city applications that are aimed at concurrently evaluating multiple solutions [13], [14], are equally insufficient in interacting with humans in a collaborative approach. Integrating the knowledge captured through computer vision-based approaches on human-infrastructure interactions with SCDT's ability to collect, store, and analyze heterogeneous city data can make community data and insights more accessible to city managers [15]. Combined with the visualization capabilities of an immersive environment, the resulting system can enable increased urban awareness for citizens, city stakeholders, and policymakers as the city grows.

In this work, we investigate integrating multi-object detection and tracking algorithms within a digital twin of the City of Columbus, GA to explore possibilities for a safer and more accessible city [11]. The Uptown Columbus digital twin, developed by the research team, is integrated with a range of city data, including ambient environment data collected by Array of Things (AoT) sensor nodes [16] installed in the local intersections of the Uptown neighborhood, as well as public surveillance camera data from the same intersections. The multi-object detection algorithm "YOLO" [17] is combined with the real-time object tracking algorithm "Deep SORT" [18] to detect multiple objects and obtain their trajectories. To assess the benefits of the proposed enhancement, we introduce a novel citizen-centric approach on community (vs. personal) exposure assessment to environmental hazards (e.g., heat stress and toxic air pollutants). This information is then used to build and test different what-if scenarios that incorporate human behavior in city decision making. For this purpose, we employ the Prophet forecasting model [19] that is trained using historical data stored in the SCDT and is able to predict urban hazard exposure.

Under consultation with the local government, our approach in this study particularly aims to address the severe problem of heat exposure in Uptown, Columbus, GA-the business center of the city-and support city managers during heat advisory periods [20].

In the next sections, a background analysis of computer vision-based studies in urban analytics as well as recent developments in SDCT is provided. The methodology for integrating object detection and tracking into SCDT is presented and evaluated through a pilot case study in two intersections in Uptown Columbus, GA. This pilot study creates the foundation of the next phases of the project, where the research team aims to enhance the system by expanding the network of intersections. Overall, this work is deemed as a first step towards integrating community dynamics into monitoring and forecasting of exposure to urban hazards and aims to increase public awareness and enable virtual assessment and analysis of what-if scenarios.

\section{Background}

\subsection{Digital twinning in smart cities}

The concept of digital twins is an emerging field of research and area of interest to the industry with applications in multiple sectors, including health, manufacturing, construction, transportation, energy, and cities [21]. The research area of smart cities has recently adopted the concept of digital twins, developing interactive platforms integrated with urban data through virtual or augmented reality [7]. A digital twin of a city in Germany focuses on visualizing heterogeneous data to engage citizens in urban planning decisions [22]. The proposed platform is focused on collaborative urban traffic planning, analyzing three different traffic scenarios through virtual reality headsets. Nonetheless, this work is not capable of interpreting human interactions. Another related study combines data sources of public transportation with social media to investigate how virtual reality can visualize big data in real-time [8]. Despite the insights on user-urban data interactions, the accuracy of the results in this work strongly relies on the level of social media use across the city.

Digital twins play a promising role in the research on city resilience and disaster management [23]. A recent study aims to enable citizens' participation in the creation of a virtual city model that raises public awareness of possible disasters [24]. Crowdsourcing data through mobile phones and photos of possible public infrastructure hazards are the main data sources. To the best of our knowledge, the promising role of SCDT in disaster management is limited in incorporating collective interactive behavior into the spatiotemporal assessments of community exposure to urban hazards [25]. An interesting approach in the field of construction progress monitoring deploys a digital twin framework that integrates object tracking and Building Information Modeling (BIM) in an immersive environment to provide construction managers with a virtual reality tool that facilitates remote progress monitoring [26]. This approach highlights the benefits of integrating object detection capabilities into an immersive environment for more effective asset management. Although promising, remotely monitoring urban operations through digital twins remains challenging. 


\subsection{Urban sensing using computer vision}

Developments in computer vision algorithms have been contributing to urban studies in the last decade, particularly in employing deep learning algorithms to both street-level images and videos, including applications such as automated detection of vehicles and humans, urban scene processing, and structural health monitoring [10].

With a focus on image processing, Zhang et al., applied a deep Convolutional Neural Network (CNN) model to uncover spatiotemporal urban mobility patterns and predict traffic flow from street-level imagery [27]. A similar approach attempts to automate urban scene understanding through detecting and analyzing transport modes and pedestrians to identify urban slums [28]. Naik et al., applied a computer vision algorithm to street-level images of urban appearances aiming to recognize and quantify changes in the urban environment [29].

Research efforts in this area have not only focused on images but also on analyzing videos for pedestrian detection and tracking, contributing to safety and traffic control. One of the first studies in this area has built a tracking system that identifies human movements in outdoor environments intending to better understand behavioral parameters compared to existing manual computations and evaluate architectural design decisions [30]. Another work proposes a classification method to automatically identify pedestrian violations to improve safety in urban intersections and reduce collisions [31]. Similarly, an object tracking algorithm was developed to automate pedestrian safety and traffic event identification and illustrate pedestrian-vehicle conflict frequencies [11]. However, these studies are limited to identifying dangerous spots or abnormalities at a certain time and place and do not store and continuously monitor urban operation. As a result, their capabilities do not enable proactive city management.

Recent advancements in edge computing, IoT technologies, and deep learning have led to real-time multi-object detection and tracking applications. A recent work proposes the integration of object tracking into IoT platforms for real-time visualization of urban data through overlay generators (heat maps in $2 \mathrm{D}$ or $3 \mathrm{D}$ graphs on images) [32]. The key to the realization of real-time object detection has initially been the widely used "YOLO" algorithm that achieved up to four times higher inference time [33]. Further technical enhancements on the real-time feasibility of multiobject tracking are provided by the algorithm "Deep SORT" [18] which can be combined with YOLO [34], [35] to count pedestrians and vehicles in real-time. In recent years, research efforts have applied this algorithm to detect pedestrian trajectories in crowded scenes in real-time [12], [36]. The characteristic that enabled YOLO as a popular algorithm in urban analytics is that it neglected the separate region proposal step which is applied in other algorithms such as Fast R-CNN [37] and Mask R-CNN[38]. This simplification, although effective, sacrifices the algorithm's accuracy, especially in detecting small objects [33], in exchange for achieving real-time detection of multiple objects in urban scenes. Focusing on understanding human behavior in public open spaces [39], a recent work applied a Mask R-CNN [38] algorithm for object tracking to understand social behavior and space utilization in public open spaces.

\subsection{Collective urban hazard exposure}

Urbanization is leading to contemporaneous increases in human activities in cities, leading to higher levels of air pollution and local overheating [40]. As a result, exposure to adverse outdoor environments is linked with millions of deaths worldwide; for example, toxic air pollutants are responsible for millions of premature deaths [41] while extremes in humidity and temperature have caused increased mortality risks [42].

In the process of mitigating the impact of urban air quality and heat stress on human health and wellbeing, exposure assessment studies have employed several tools and methods. Recently, researchers in the field have investigated the role of individual travel patterns in their exposure assessments to overcome the limitations imposed by traditional static (vs. dynamic) approaches that take collective exposure of the population into account based on their home locations [43]. Current dynamic approaches such as the one proposed in [44], are also limited as they either employ mobile phone data to locate human movements in the city and measure their NO2 exposure, or incorporate the dynamics of population into large scale (e.g., nationwide) exposure estimations [45]. These approaches are focused on understanding exposure at a city-wide scale (as opposed to local community scales) and are generally limited due to data unavailability and the high cost nature of the experiment [44]. To enhance personal exposure assessments, research has mainly considered sociodemographic characteristics and activity patterns of individuals captured through wearable portable environmental sensors used by local volunteers [46]. Similarly, outdoor thermal comfort assessments use wearable sensors and incorporate physical, physiological, psychological, and social behavior of users [48] to evaluate pedestrian level urbanmicroclimate patterns in real-time [47]. The main drawbacks of these approaches stem from the small sample size, consisting of mostly healthy adults, and the cost of the portable sensors. 
To overcome the above limitations, we introduce a novel citizen-centric approach for assessing collective (vs. personal) urban hazard exposure in cities. The proposed methodology is focused on providing city managers with the ability to monitor exposure in realtime and make decisions that could shield citizens from hazardous levels of heat stress and transform overheated public spaces.

\subsection{Heat exposure in Columbus, GA}

According to the Columbus Health Department [20], heat stress management is deemed to be a major problem for city managers, who are often challenged to convince citizens of the severity of the problem, notwithstanding that heat stroke is a leading cause of death among children during heatwaves. The mayor of Columbus in cooperation with the Safe Kids organization has highlighted the need to understand that children cannot handle the heat like an adult [48]. Locating and forecasting community heat stress exposure can be helpful for city managers to manage the problem more effectively (i.e., for local interventions such as shading points or hydration stations). While diffusing the information through the immersive environment of the Uptown Columbus digital twin could increase citizens' awareness about the severity of the problem.

As a part of an ongoing SCDT deployment, a pilot study was designed for the city of Columbus to demonstrate how this approach can contribute to proactive city management. The objectives of this work are summarized as follows: (1) Devise a novel exposure assessment method using computer vision algorithms to capture community dynamics in the city; (2) Deploy a novel citizen-centric approach for assessing collective urban hazard exposure and locating hazardous spots; (3) Build a forecasting model that is able to predict upcoming urban hazards (i.e., dangerous heatwave exposures); (4) Provide monitoring capability using an immersive environment where city managers can visualize what-if scenarios, while citizens can engage to become aware and provide feedback; (5) Equip the city with an asset for proactive city management and early identification of hazards.

\section{Methodology}

\subsection{Smart Uptown Digital Twin}

The City of Columbus, Georgia was used as a testbed for this study, which is part of an ongoing project to create a digital twin of Uptown Columbus. The Uptown district is the business center of Columbus, a place where large public events take place, with an increasing need for improved public safety and security. The first phase of the project, which includes two central intersections of Broadway-on $10^{\text {th }}$ Street and $11^{\text {th }}$ Street - in the Uptown neighborhood, is the focus of this study (Figure 1). In the next phases, sensors will be installed on more intersections throughout Uptown.

The Smart Uptown Digital Twin (Figure 2) enables users to virtually explore the neighborhood while accessing location-specific data that is continuously

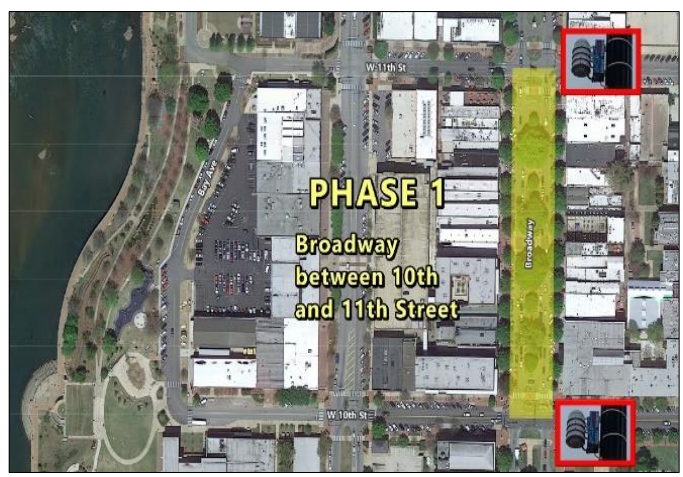

Figure 1: Location of sensor-instrumented intersections (e.g., AoT sensor nodes) in Uptown Columbus, GA-Project Phase 1.

being streamed from the AoT [16] sensor nodes, The AoT nodes collect a range of urban data including climate (humidity and temperature) and air pollution (five toxic air pollutants) data as depicted in Table 1.

Table 1: AoT sensor metrics

\begin{tabular}{|l|l|}
\hline Sensor Branch & Metrics \\
\hline Climate & Humidity, Temperature \\
\hline \multirow{2}{*}{ Air pollution } & $\begin{array}{l}\text { Sulfur Dioxide (SO2), Nitrogen } \\
\text { Dioxide (NO2), Ozone (O3), } \\
\text { Particulate Matter (PM2.5) }\end{array}$ \\
\hline
\end{tabular}

\subsection{Passersby detection and tracking}

In order to integrate object detection and tracking into the SCDT platform, we employed the multi-object detection algorithm YOLOv3 and the real-time object tracking algorithm Deep SORT. The combined method was implemented in Python using the OpenCV library and TensorFlow, and the training process was based on pre-trained datasets.

YOLOv3 stands for "You Only Look Once" and is founded on a convolutional neural network (CNN) [17]. The dominance of the algorithm is based on its ability to predict classes' labels and detect locations of objects at the same time. The following steps have been applied to run the YOLOv3 algorithm on the surveillance camera data at each intersection [33]: (1) Divide an image into regions and predict bounding boxes and class 


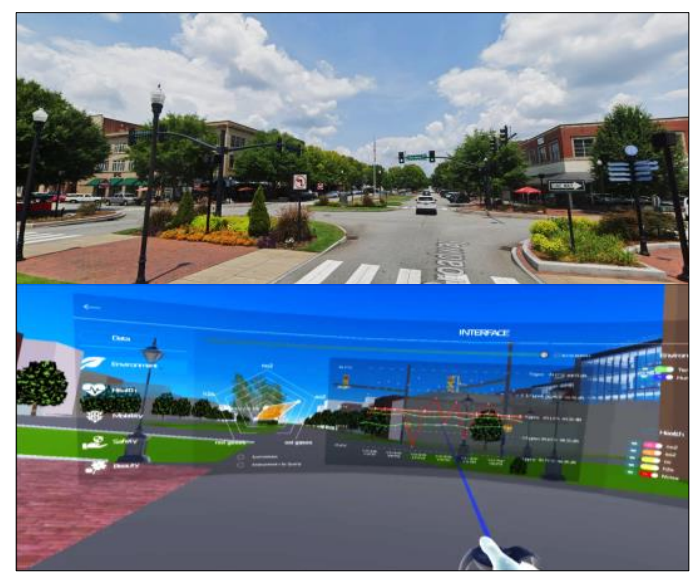

Figure 2: Smart Uptown Digital Twin.

probabilities for every region; (2) Predict confidence score of every bounding box; and (3) Filter the bounding boxes excluding some of them if the confidence score is low or there is another bounding box with a higher confidence score in the same region.

Besides locating multiple objects, tracking an object constitutes a different problem since detection algorithms do not maintain object persistence between video frames. In our approach, we used the Deep SORT algorithm, which builds upon object detection algorithms such as YOLOv3 to maintain the locations of the detected objects. The success of Deep SORT in tracking multiple objects in real-time stems from the Kalman filter that factors in the noise of the detection while using prior state in predicting a good fit for bounding boxes [18]. The object tracker offers additional capabilities such as crowd counting and jaywalker detection [34].

In the first phase of the project, the two algorithms were implemented on the surveillance camera data located at two intersections of Broadway-on $10^{\text {th }}$ Street and $11^{\text {th }}$ Street - as illustrated in Figure 1. For the purpose of our pilot study, we detect and quantify the passersby (i.e. pedestrians, bikes, etc.) crossing these intersections to discover and understand the community dynamics of the area. Figure 3 shows a snapshot from

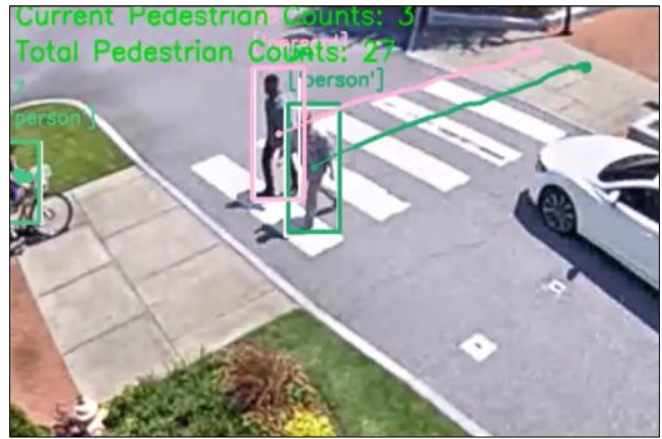

Figure 3: Detection and tracking of passersby -intersection of Broadway and $10^{\text {th }}$ Street. detecting and tracking passersby during the implementation of the two algorithms. The information including the obtained trajectories of the passersby will be analyzed in the next phases of the project to discover issues related to pedestrians' safety and interaction with vehicles (e.g., jaywalking, wheelchair accessibility, bike violations, etc.).

\section{Application and Results}

\subsection{Heat stress monitoring}

In developing an approach that enables monitoring and forecasting of collective urban hazard exposure, we employed the aforementioned object detection and tracking algorithms to capture the community's spatiotemporal exposure rates at the $10^{\text {th }}$ Street $\& 11^{\text {th }}$ Street intersections with Broadway.

For the purpose of this study, we considered the following measures: We implemented the pilot study for one week from Sunday, August $16^{\text {th }}, 2020$ to Saturday, August $22^{\text {nd }}, 2020$ to assess how the community dynamics in these intersections influence the rates of heat exposure during a typical summer week. Temperature and humidity are known to be primarily responsible for heat-related health problems such as low-energy level, emotional problems, low life satisfaction, and even death due to heatstroke [49]. To monitor the level of heat stress, we used the well-known Temperature-Humidity Index (THI) [50], which combines temperature and humidity measures to classify the stress levels from "very warm" to "extremely hot" as follows:

$$
T H I=t-(0.55-0.0055 R H)(t-58)
$$

where $t$ represents air temperature and $\mathrm{RH}$ equals relative humidity (\%). Figure 4 shows the THI levels for both intersections during the study period, revealing that citizens are more likely to be exposed to heat stress at the intersection on $10^{\text {th }}$ Street compared to $11^{\text {th }}$ Street. Even during nighttime, $10^{\text {th }}$ Street exceeds the stress level "Hot" and in the first days reaches the "Very Hot" level. It also worth mentioning that during Sunday, August $16^{\text {th }}$ between $9 \mathrm{pm}$ and $10 \mathrm{pm}$, the THI at the $10^{\text {th }}$ Street intersection reached the level of 108 ("Very Hot") while during the same time period the $11^{\text {th }}$ Street intersection was 85 ("Very Warm"), amounting to the largest reported difference during that week.

Some of the reasons that can cause one intersection to be more exposed to heat compared to the other include, urban configurations such as shading or greenery, more through traffic causing local 
overheating, obstructed air flow due to urban morphology, etc.

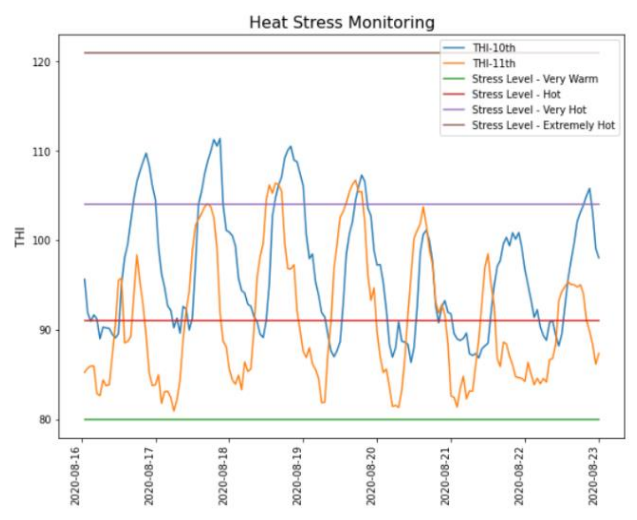

Figure 4: Temperature - Humidity Index (THI) from Aug 16-22, 2020 for the two intersections of Broadway on $10^{\text {th }}$ Street and $11^{\text {th }}$ Street.

\subsection{Passersby monitoring for heat exposure discovery}

The object detection and tracking was applied to daily ( 24 hour) camera feeds over the entire study period to quantify the number of passersby and combine it with the level of heat exposure at each intersection. Figure 5 illustrates the total number of passersby that crossed each intersection every 4 hours within the duration of the study. The occupancy of the $10^{\text {th }}$ Street intersection on weekdays seems to be similar (or sometimes higher) than the $11^{\text {th }}$ Street intersection for this particular week. However, the number of passersby that crossed the $11^{\text {th }}$ Street intersection is higher during the weekend. More precisely, on Saturday, August $22^{\text {nd }}$, the occupancy of the $11^{\text {th }}$ Street intersection might indicate a particular event happening in the area. From 12 am to 4 pm on the same day, 1624 passersby crossed $11^{\text {th }}$ Street while 425

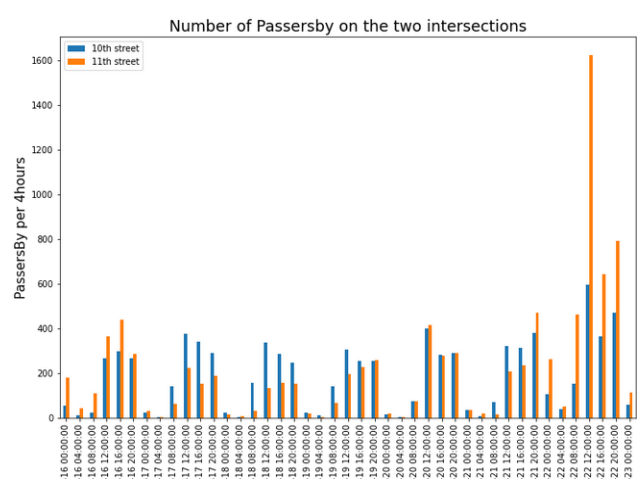

Figure 5: Total number of Passersby in both intersections of Broadway on $10^{\text {th }}$ Street and $11^{\text {th }}$ Street for one week crossed $10^{\text {th }}$ Street, reaching the highest values for the entire week. In total, $11^{\text {th }}$ Street seems to have received a substantially higher number of passersby, which suggests a potentially higher rate of urban hazard exposure. Overall, it is evident that certain time periods and locations involve higher risks for heat stress when we factor in the number of heat-exposed people.

Although the THI (Figure 4) can inform us about the level of heat stress throughout the day, it does not account for the level of human exposure, and this information does not yet represent a collective rate of exposure for the community. As a first step towards this realization, we introduce Passersby Weighted Exposure (PWE) THI as follows, which combines heat stress measurements with a quantified measure of human exposure at any given time. The daily total PWE THI in intersection $i$ is calculated as follows:

$$
P W E_{T H I, i}=\frac{1}{P} \sum_{t=1}^{24} T H I_{i, t} \cdot P_{i, t}
$$

where $P$ is the total number of intersections (just two for this phase), $P_{i, t}$ and $T H I_{i, t}$ indicate the number of passersby and the THI value in intersection $i$ at time $t$, respectively.

As expected, heat stress exposure is higher on Saturday, August $22^{\text {nd }}$, considering that the number of passersby that crossed the intersection is higher on that day (Figure 6). Comparing Figure 6 to Figure 4, our approach establishes a new way of assessing collective heat stress exposure and outdoor comfort based on the extent of occupancy of public spaces by the community.

\subsection{What-if scenario-based decision making}

The Smart Uptown Digital Twin is expected to store and analyze historical information from daily city operations in order to generate and visualize what-if

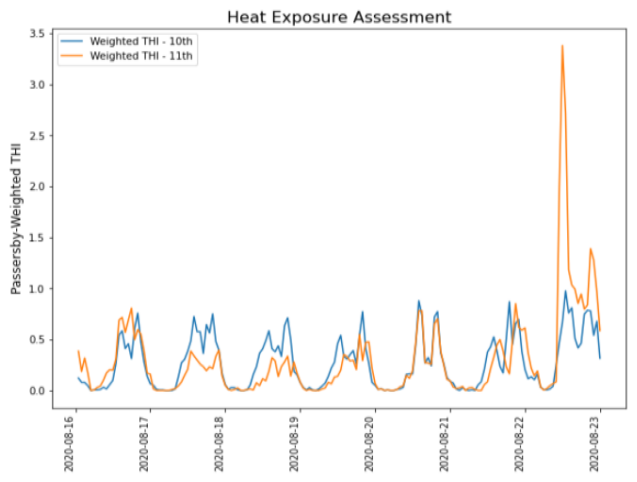

Figure 6: Passersby-Weighted THI from Aug 16-23, 2020 for the two intersections of Broadway on $10^{\text {th }}$ Street and $11^{\text {th }}$ Street. 
scenarios that will help city managers make proactive decisions for preventing people from urban hazard exposure early on. To this end, we deployed a forecasting model that is able to make predictions based on heat stress exposure data.

In particular, we used Facebook's time series forecasting procedure owing to its robustness in handling missing data and outliers well [19]. For the pilot study, PWE THI measurements are used to train the model and predict next-day 24-hour heat exposure for both intersections.

Figure 7 presents the forecasting model for the intersection on $10^{\text {th }}$ Street. The black dots represent the real data points, the blue line is the prediction model, and the shaded blue areas constitute the upper and lower confidence bounds of the model. In Figure 8, the forecasted data is compared with the real data for PWE THI measurements of the $10^{\text {th }}$ Street intersection. It is expected that by storing and analyzing further historical data, both the accuracy of the predictions and the capability for increasing the prediction horizon will be

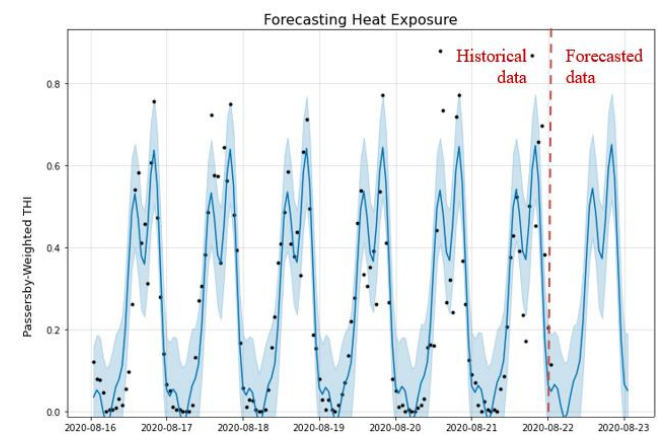

Figure 7: Forecasting model on $10^{\text {th }}$ Street improved.

To explain how the integration of the proposed model with SCDT can be useful for city managers and citizens, the next-day predictions of both intersections are presented in Figure 9. Based on the historical data,

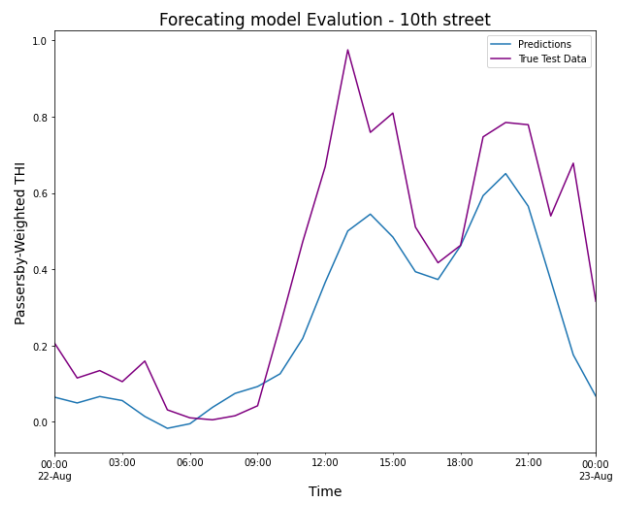

Figure 8: Forecasting model evaluation: Real vs. predicted PWE THI on $10^{\text {th }}$ Street. we can predict that a higher heat exposure rate is more likely to happen on $10^{\text {th }}$ Street than on $11^{\text {th }}$ Street. This information can be useful during the heat advisory period in Columbus, GA to support certain locations throughout the city with hydration points, fans or additional shading.

Furthermore, considering that Uptown Columbus is the business center where big events usually take place, the ability to predict the heat stress exposure can be a useful utility for optimal scheduling of big events. For example, an example of a civil unrest in the same intersection (a Black Lives Matter protest that occurred on $10^{\text {th }}$ Street) poses interesting research questions regarding the management of similar events when many people are exposed to heat stress, toxic air pollutants, and other urban hazards.

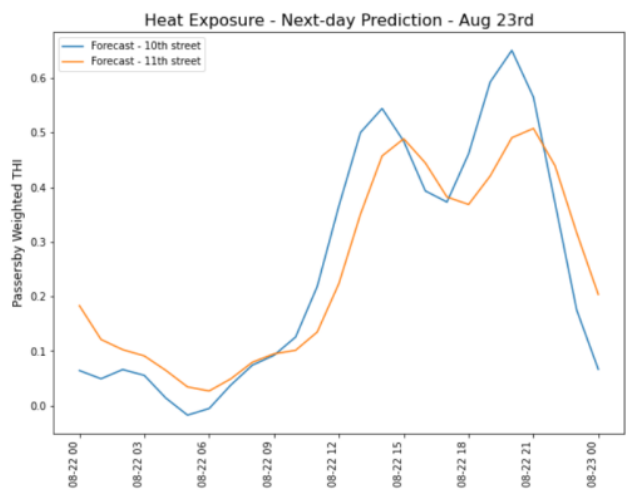

Figure 9: Next-day predictions of PWE THI for both intersections of Broadway on $10^{\text {th }}$ Street and $11^{\text {th }}$ Streets.

\section{Discussion and Conclusions}

In this study, we introduce a computer vision-based monitoring and forecasting approach for Smart City Digital Twins (SCDT) that enables integrating collective community behavior into the assessments of exposure to urban hazards across time and space. Our approach highlights how SCDT can make community dynamics more accessible to city managers. This informs city managers about the spatiotemporal fluctuations in the level of exposure of the community to adverse thermal comfort conditions and other hazards. City managers can identify hidden or forthcoming abnormalities (e.g., an increasing trend of heat stress is more harmful during busy times) and thus, establish SCDT as an asset for the proactive management of the city. The proposed method is supported by a forecasting model that is able to predict heat stress community exposure throughout the city. The ability of the SCDT to predict and monitor hazard exposure will be the foundation for designing and 
visualizing what-if scenarios of upcoming urban hazards. As the SCDT stores more historical data, the system becomes more capable of uncovering and predicting the variations in the rates of community exposure to urban hazards.

The proposed approach to urban hazard exposure assessment incorporates collective community behavior and interactions into city management and decision making, whereas previous approaches are limited to assessing personal exposure of individuals and primarily rely on the availability of mobile phone data or costly wearable sensors. In the next phases of the project, we aim to further quantify the vehicles using video sources, as well as determining levels of greenery and buildings' heights to explore their impact. These insights can be particularly useful for urban planners in implementing regional and local interventions, including urban design (i.e., shading points, planting trees) and management of areas that are more exposed to heatwaves (e.g., installing hydration points, fans, or additional shading). This is an ongoing study seeks to expand the monitoring and forecasting capabilities of cities in general, and more specifically collective community exposure to urban hazards. This will, in turn, empower city management and enhance what-if scenario analyses with a more citizen-centric approach.

\section{Acknowledgements}

This material is based upon work supported by the Georgia Smart Communities Challenge program and the National Science Foundation under Grant No. 1837021. Any opinions, findings, and conclusions or recommendations expressed in this material are those of the authors and do not necessarily reflect the views of the National Science Foundation. The authors would like thank the Columbus Consolidated Government (CCG) for their collaboration and support.

\section{References}

[1] United Nations Department of Economic and Social Affairs, World Population Prospects 2019. 2019.

[2] United Nations, "The World 's Cities in 2018," World's Cities 2018 - Data Bookl. (ST/ESA/ SER.A/417), 2018.

[3] P. Marana et al., "Towards a resilience management guideline - Cities as a starting point for societal resilience," Sustain. Cities Soc., vol. 48, 2019.

[4] Y. Sun, H. Song, A. J. Jara, and R. Bie, "Internet of Things and Big Data Analytics for Smart and Connected Communities," IEEE Access, vol. 4, pp. 766-773, 2016.

[5] J. Viitanen and R. Kingston, "Smart cities and green growth: Outsourcing democratic and environmental resilience to the global technology sector," Environ. Plan. A, vol. 46, no. 4, pp. 803-819, 2014.

[6] M. U. Bers and C. Chau, "Fostering civic engagement by building a virtual city," J. Comput. Commun., vol. 11 , no. 3, pp. 748-770, 2006.

[7] N. Mohammadi and J. E. Taylor, "Smart city digital twins," in Proceedings - IEEE Symposium Series on Computational Intelligence, SSCI 2017, pp. 1-5, 2018.

[8] J. Perhac et al., "Urban fusion: Visualizing urban data fused with social feeds via a game engine," in Proceedings - $21^{\text {st }}$ International Conference Information Visualisation, iV 2017, pp. 312-317, 2017.

[9] X. Kong, X. Liu, B. Jedari, M. Li, L. Wan, and F. Xia, "Mobile Crowdsourcing in Smart Cities: Technologies, Applications, and Future Challenges," IEEE Internet Things J., vol. 6, no. 5, pp. 8095-8113, 2019.

[10] M. R. Ibrahim, J. Haworth, and T. Cheng, "Understanding cities with machine eyes: A review of deep computer vision in urban analytics," Cities, vol. 96, 2020.

[11] M. Zaki, T. Sayed, A. Tageldin, and M. Hussein, "Application of computer vision to diagnosis of pedestrian safety issues," Transp. Res. Rec., no. 2393, pp. 75-84, 2013.

[12] J. Barthélemy, N. Verstaevel, H. Forehead, and P. Perez, "Edge-computing video analytics for real-time traffic monitoring in a smart city," Sensors (Switzerland), vol. 19, no. 9, 2019.

[13] A. Harris, J. Stovall, and M. Sartipi, "MLK Smart Corridor: An Urban Testbed for Smart City Applications," in Proceedings - IEEE International Conference on Big Data, Big Data 2019, pp. 35063511, 2019.

[14] V. M. Larios, L. Gomez, O. B. Mora, R. Maciel, and N. Villanueva-Rosales, "Living labs for smart cities: A use case in Guadalajara City to foster innovation and develop citizen-centered solutions," in Proceedings $2^{\text {nd }}$ IEEE International Smart Cities Conference: Improving the Citizens Quality of Life, ISC2 2016, 2016.

[15] N. Mohammadi and J. Taylor, "Knowledge Discovery in Smart City Digital Twins," in Proceedings - 53 ${ }^{\text {rd }}$ Hawaii International Conference on System Sciences, 2020.

[16] C. E. Catlett, P. H. Beckman, R. Sankaran, and K. K. Galvin, "Array of things: A scientific research instrument in the public way," in Proceedings $-2^{\text {nd }}$ International Workshop on Science of Smart City Operations and Platforms Engineering, in partnership with Global City Teams Challenge, SCOPE 2017, pp. 26-33, 2017.

[17] J. Redmon and A. Farhadi, "YOLO v.3," Tech Rep., 2018.

[18] N. Wojke, A. Bewley, and D. Paulus, "Simple online and realtime tracking with a deep association metric," in Proceedings - International Conference on Image Processing, ICIP, vol. 2017-Sept, pp. 3645-3649, 2018.

[19] S. J. Taylor and B. Letham, "Business Time Series Forecasting at Scale," PeerJ Prepr. 5e3190v2, vol. 35, no. 8, pp. 48-90, 2017.

[20] WTVM, "Columbus health official urge safety during heat advisory," 2019. [Online]. Available: https://www.wtvm.com/2019/09/09/columbus-healthofficial-urge-safety-during-heat-advisory/.

[21] A. Rasheed, O. San, and T. Kvamsdal, "Digital twin: Values, challenges and enablers from a modeling perspective," IEEE Access, 2020. 
[22] F. Dembski, U. W. Ssner, and C. Yamu, "Digital twin, virtual reality and space syntax: Civic engagement and decision support for smart, sustainable cities," in Proceedings - $12^{\text {th }}$ International Space Syntax Symposium, 2019.

[23] R. Desroches and J. E. Taylor, "The promise of smart and resilient cities," Bridge, vol. 48, no. 2, pp. 13-20, 2018.

[24] Y. Ham and J. Kim, "Participatory Sensing and Digital Twin City: Updating Virtual City Models for Enhanced Risk-Informed Decision-Making," J. Manag. Eng., vol. 36, no. 3, p. 04020005, 2020.

[25] C. Fan, C. Zhang, A. Yahja, and A. Mostafavi, "Disaster City Digital Twin: A vision for integrating artificial and human intelligence for disaster management," Int. J. Inf. Manage., 2019.

[26] F. Pour Rahimian, S. Seyedzadeh, S. Oliver, S. Rodriguez, and N. Dawood, "On-demand monitoring of construction projects through a game-like hybrid application of BIM and machine learning," Autom. Constr., vol. 110, 2020.

[27] F. Zhang, L. Wu, D. Zhu, and Y. Liu, "Social sensing from street-level imagery: A case study in learning spatio-temporal urban mobility patterns," ISPRS J. Photogramm. Remote Sens., vol. 153, pp. 48-58, 2019.

[28] M. R. Ibrahim, J. Haworth, and T. Cheng, "URBAN-i: From urban scenes to mapping slums, transport modes, and pedestrians in cities using deep learning and computer vision," Environ. Plan. B Urban Anal. City Sci., 2019.

[29] N. Naik, S. D. Kominers, R. Raskar, E. L. Glaeser, and C. A. Hidalgo, "Computer vision uncovers predictors of physical urban change," Proc. Natl. Acad. Sci. U. S. A., vol. 114, no. 29, pp. 7571-7576, 2017.

[30] W. Yan and D. A. Forsyth, "Learning the behavior of users in a public space through video tracking," in Proceedings - Seventh IEEE Workshop on Applications of Computer Vision, WACV 2005, pp. 370-377, 2005.

[31] M. Zaki, T. Sayed, K. Ismail, and F. Alrukaibi, "Use of computer vision to identify pedestrians' nonconforming behavior at urban intersections," Transp. Res. Rec., no. 2279, pp. 54-64, 2012.

[32] J. Stovall, A. Harris, A. O'Grady, and M. Sartipi, "Scalable Object Tracking in Smart Cities," in Proceedings - 2019 IEEE International Conference on Big Data, Big Data 2019, pp. 3813-3819, 2019.

[33] J. Redmon, S. Divvala, R. Girshick, and A. Farhadi, "You only look once: Unified, real-time object detection," in Proceedings - IEEE Computer Society Conference on Computer Vision and Pattern Recognition, vol. 2016Decem, pp. 779-788, 2016.

[34] Y. Le Yang and W. W. Gao, "A method of pedestrians counting based on deep learning," in Proceedings 2019 IEEE $3^{\text {rd }}$ International Conference on Electronic Information Technology and Computer Engineering, EITCE 2019, pp. 2010-2013., 2019.

[35] S. Kapania, D. Saini, S. Goyal, N. Thakur, R. Jain, and P. Nagrath, "Multi object tracking with UAVs using deep SORT and YOLOv3 RetinaNet detection framework," in Proceeding - $1^{\text {st }}$ ACM Workshop on Autonomous and Intelligent Mobile Systems, 2020.
[36] S. Nagaraj, B. Muthiyan, S. Ravi, V. Menezes, K. Kapoor, and H. Jeon, "Edge-based street object detection," in Proceedings - 2017 IEEE SmartWorld Ubiquitous Intelligence and Computing, Advanced and Trusted Computed, Scalable Computing and Communications, Cloud and Big Data Computing, Internet of People and Smart City Innovation, SmartWorld/SCALCOM/UIC/ATC/CBDCom/IOP/SCI 2017, pp. 1-4, 2018.

[37] R. Girshick, "Fast R-CNN," in Proceedings - IEEE International Conference on Computer Vision, vol. 2015 Inter, pp. 1440-1448, 2015.

[38] K. He, G. Gkioxari, P. Dollar, and R. Girshick, "Mask RCNN," in Proceedings - IEEE International Conference on Computer Vision, vol. 2017-Octob, pp. 2980-2988, 2017.

[39] P. Sun, R. Hou, and J. P. Lynch, “A Computer vision framework for human user sensing in public open spaces," in Proceedings - $1^{\text {st }}$ ACM Workshop on DeviceFree Human Sensing, pp. 27-30, 2019.

[40] S. M. Willers et al., "High resolution exposure modelling of heat and air pollution and the impact on mortality," Environ. Int., vol. 89-90, pp. 102-109, 2016.

[41] WHO, "7 Million Premature Deaths Annually Linked to Air Pollution.," World Health Organization., 2019. .

[42] J. Paravantis, M. Santamouris, C. Cartalis, C. Efthymiou, and N. Kontoulis, "Mortality associated with high ambient temperatures, heatwaves, and the urban heat island in Athens, Greece," Sustain., vol. 9, no. 4, 2017.

[43] S. Steinle, S. Reis, and C. E. Sabel, "Quantifying human exposure to air pollution-Moving from static monitoring to spatio-temporally resolved personal exposure assessment," Science of the Total Environment, vol. 443. pp. 184-193, 2013.

[44] B. Dewulf et al., "Dynamic assessment of exposure to air pollution using mobile phone data," Int. J. Health Geogr., vol. 15, no. 1, 2016.

[45] B. Chen, Y. Song, T. Jiang, Z. Chen, B. Huang, and B. $\mathrm{Xu}$, "Real-time estimation of population exposure to PM2.5 using mobile- and station-based big data," Int. J. Environ. Res. Public Health, vol. 15, no. 4, 2018.

[46] L. Liang, P. Gong, N. Cong, Z. Li, Y. Zhao, and Y. Chen, "Assessment of personal exposure to particulate air pollution: The first result of City Health Outlook (CHO) project," BMC Public Health, vol. 19, no. 1, 2019.

[47] I. Pigliautile, G. Marseglia, and A. L. Pisello, "Investigation of $\mathrm{CO} 2$ variation and mapping through wearable sensing techniques for measuring pedestrians' exposure in urban areas," Sustain., vol. 12, no. 9, p. 3936, May 2020.

[48] WTVM, "Heat safety discussion in Columbus focuses on preventing heat stroke," 2020.

[49] B. Tawatsupa, V. Yiengprugsawan, T. Kjellstrom, S. A. Seubsman, and A. Sleigh, "Heat stress, health and wellbeing: Findings from a large national cohort of Thai adults," BMJ Open, 2012.

[50] R. G. Steadman, "The assessment of sultriness. Part I. A temperature-humidity index based on human physiology and clothing science.," J. Appl. Meteorol., vol. 18 , no. 7, pp. 861-873, 1979. 